\title{
Evidence for Plasmid-mediated Restriction-Modification in Mycobacterium avium intracellulare
}

\author{
By JACK T. CRAWFORD, 1,2* M. DONALD CAVE ${ }^{3}$ AND \\ JOSEPH H. BATES ${ }^{1,4}$ \\ ${ }^{1}$ Medical Research, Veterans Administration Medical Center, Little Rock, Arkansas 72206, \\ U.S.A. \\ 2,3,4 Departments of Microbiology and Immunology ${ }^{2}$, Anatomy $^{3}$ and Medicine $e^{4}$, University of \\ Arkansas for Medical Sciences, Little Rock, Arkansas 72201, U.S.A.
}

(Received 8 April 1981)

\begin{abstract}
Mycobacterium avium intracellulare strain LR25 carries three plasmids with molecular weights of $11.2,18.3$ and $107 \times 10^{6}$ as determined by electron microscopy. A number of phages propagated on Mycobacterium smegmatis ATCC 607 were tested for their ability to infect strain LR25. Phage JF2 gave an efficiency of plating of $10^{-4}$ on strain LR25, but phage JF2 propagated on strain LR25 infected strain LR25 and M. smegmatis with equal high efficiency. This indicated the presence of a restriction-modification (R-M) system in strain LR25 that was not present in $M$. smegmatis. Strain LR25 was grown in the presence of acriflavine to eliminate the plasmids and tested for sensitivity to phage JF2. One of forty colonies was found to be R-M-deficient. This strain, designated strain LR163, lacks the three plasmids present in strain LR25. The results indicate that the $R-M$ system is plasmid-coded. Strain LR163 was sensitive to several phages to which strain LR25 was resistant and for which we were unable to isolate modified phage. This suggests that some plasmid-coded function in addition to restriction is involved. An R-M system was also demonstrated in $M$. avium intracellulare strain LR131 using phage JF1. This strain does not carry plasmids.
\end{abstract}

\section{INTRODUCTION}

Although there have been extensive studies on the physiology of mycobacteria, knowledge of their genetics is rudimentary. In a previous paper we described a procedure for gentle lysis of mycobacteria and isolation of DNA and reported the existence of plasmids in Mycobacterium avium intracellulare (Crawford \& Bates, 1979). Because plasmids can be readily defined by physical means they offer an excellent model for developing genetic analysis in mycobacteria. In addition, plasmid-coded functions may prove to be important in the physiology of mycobacteria. However, determining the function of a newly identified plasmid can be difficult, especially in slowly growing organisms like mycobacteria. We are developing a phage typing scheme for $M$. avium intracellulare, and this work has revealed the presence of restriction-modification $(R-M)$ systems in these organisms. This paper reports the demonstration of an R-M system in strain LR25, a strain that carries three plasmids, and the isolation of a cured $\mathbf{R}-\mathbf{M}$-deficient derivative.

\section{METHODS}

Media. Mycobacterium avium intracellulare strains were cultured in 7H9-Av broth containing Middlebrook 7H9 broth, $0.5 \%(\mathrm{w} / \mathrm{v})$ glycerol and Dubos oleic acid/albumin enrichment. Dubos oleic acid/albumin agar plates and soft agar containing Middlebrook $7 \mathrm{H} 9$ broth, $0.05 \%(\mathrm{w} / \mathrm{v})$ protease peptone, $0.2 \%(\mathrm{w} / \mathrm{v})$ glycerol and $0.7 \%$ $(w / v)$ Noble agar were used for soft agar overlay technique. Mycobacterium smegmatis was cultured in NB3 broth 
containing $0.8 \%(\mathrm{w} / \mathrm{v})$ nutrient broth, $0.5 \%(\mathrm{w} / \mathrm{v}) \mathrm{NaCl}$ and $0.5 \mathrm{~mm}-\mathrm{CaCl}_{2}, \mathrm{pH} \mathrm{7.0.} \mathrm{For} \mathrm{soft} \mathrm{agar} \mathrm{overlay}$ technique, the bottom agar consisted of $\mathrm{NB} 3$ plus $1 \mathrm{~mm}-\mathrm{MgSO}_{4}, 1.6 \%(\mathrm{w} / \mathrm{v})$ glycerol, and $1.1 \%(\mathrm{w} / \mathrm{v})$ Noble agar, and the soft agar contained NB3 plus $0.6 \%(w / v)$ Noble agar. All components of media were obtained from Difco.

Bacteria and phage. Mycobacterium avium intracellulare strain LR25 was isolated from a patient at this hospital and was judged to be a pulmonary pathogen by the clinician in charge. Strain LR131 and other M. avium intracellulare strains were obtained from J. K. McClatchy, National Jewish Hospital, Denver, Colo., U.S.A. Mycobacterium smegmatis ATCC 607 was from our laboratory stock.

Phages JF1, JF2, JF3 and JF4 were isolated in this laboratory from barnyard soil. Other phages were obtained from various sources. Phages were propagated and their titres determined on $M$. smegmatis using the soft agar overlay technique. For plaque assays, $M$. avium intracellulare strains were cultured in $7 \mathrm{H} 9$-Av broth in shaken flasks to an $\boldsymbol{A}_{600}$ of about $\mathbf{0 . 8}$. Soft agar overlays were inoculated with one drop (about $0.05 \mathrm{ml}$ ) of culture. Further details of the phages and the technique for plaque assays with $M$. avium intracellulare strains will be presented elsewhere. Phages are designated by the phage strain followed by the propagating host, e.g. JF2-607.

Isolation of cured strains. Strain LR25 was cultured in 7H9-Av broth to mid-exponential phase and then inoculated into a series of tubes containing twofold serial dilutions of neutral acriflavine (Sigma) in 7H9-Av broth. The tubes were incubated for $48 \mathrm{~h}$ at $37^{\circ} \mathrm{C}$. The cultures were diluted 100 -fold into 7H9-Av broth and incubated for an additional $72 \mathrm{~h}$. The culture treated with $12.5 \mu \mathrm{g}$ acriflavine $\mathrm{ml}^{-1}$ showed only slight growth inhibition compared to a control; it was diluted and plated on Dubos oleic acid/albumin plates. After a 2 week incubation, isolated colonies were transferred to $7 \mathrm{H} 9$-Av broth and incubated for $7 \mathrm{~d}$. Each culture was tested for sensitivity to phages JF2-607 and JF2-LR25.

Isolation of plasmid DNA. Preparation of sodium dodecyl sulphate/salt cleared lysates was performed as previously described (Crawford \& Bates, 1979) except that the cells were cultured in 7H9-Av broth and ampicillin $\left(0.1 \mathrm{mg} \mathrm{ml}^{-1}\right)$ was added in addition to cycloserine to sensitize cells to detergent lysis. Plasmid DNA was prepared by $\mathrm{CsCl}$ /ethidium bromide equilibrium gradient centrifugation using a fixed angle Beckman Type 50.2 or Type 75 Ti rotor. The satellite band was removed and re-banded in a swinging bucket Beckman SW65Ti rotor. The sample was extracted with 2-propanol and dialysed against TES buffer (50 mM-NaCl, $5 \mathrm{~mm}$-EDTA, $30 \mathrm{mM}$-Tris, pH 8.0).

Electrophoresis. Agarose gels $(20 \times 20 \times 0.5 \mathrm{~cm})$ were run in a horizontal gel electrophoresis apparatus using either Tris/acetate/EDTA (Johnson \& Grossman, 1977) or Tris/borate/EDTA (Meyers et al., 1976) buffer. Gels were run at $150 \mathrm{~V}$ for $12 \mathrm{~h}$. Gels were stained with ethidium bromide and illuminated with a short wavelength ultraviolet transilluminator (Ultra-Violet Products, San Gabriel, Calif., U.S.A.) for photography.

Electron microscopy. DNA was spread according to the formamide modification of the Kleinschmidt procedure (Davis et al., 1971). For measurements of contour length, DNA was spread in $70 \%(\mathrm{w} / \mathrm{v})$ formamide with double-stranded $\phi$ X-174 DNA as an internal standard. Molecules were picked up on Parlodion-coated grids, treated with $50 \mu \mathrm{M}$ aqueous uranyl acetate and air dried. The grids were rotary-shadowed with platinum/palladium (4:1) at an angle of $7 \circ$. Electron micrographs were taken in a Siemens 1 -A electron microscope at an initial magnification of 8000 . The negatives were enlarged to a final magnification of 50000 . Contour lengths were measured with a map measurer and converted to molecular weights by comparison with adjacent $\phi \mathrm{X}-174$ molecules.

\section{RESULTS}

\section{Plasmids of strain LR25}

Analysis of DNA from strain LR25 revealed three plasmids, designated pLR1, pLR2 and pLR3 (Fig. 1). When crude polyethylene glycol-precipitated DNA was subjected to electrophoresis in Tris/acetate buffer the intermediate-sized plasmid (pLR2) was often obscured by chromosomal DNA fragments, and this plasmid was apparently missed in our preliminary work (Crawford \& Bates, 1979). In Tris/borate buffer all three plasmids migrated more slowly than the chromosomal DNA (results not shown). This suggests that the use of these two buffers when screening crude DNA samples would ensure that all plasmids are resolved. Plasmid DNA was examined by electron microscopy to verify that the DNA was circular (Fig. 2). The molecular weights of the plasmids were calculated to be ( \pm S.D.): pLR1, $11.19 \pm 1.06 \times 10^{6}$ (46 molecules measured); pLR2, $18.33 \pm 2.02 \times 10^{6}(23$ molecules); and pLR3, 106.9 $\pm 6 \times 10^{6}$ (41 molecules). Our previous estimate of the size of pLR1 was based on a comparison with an incorrectly identified marker plasmid and was inaccurate. 


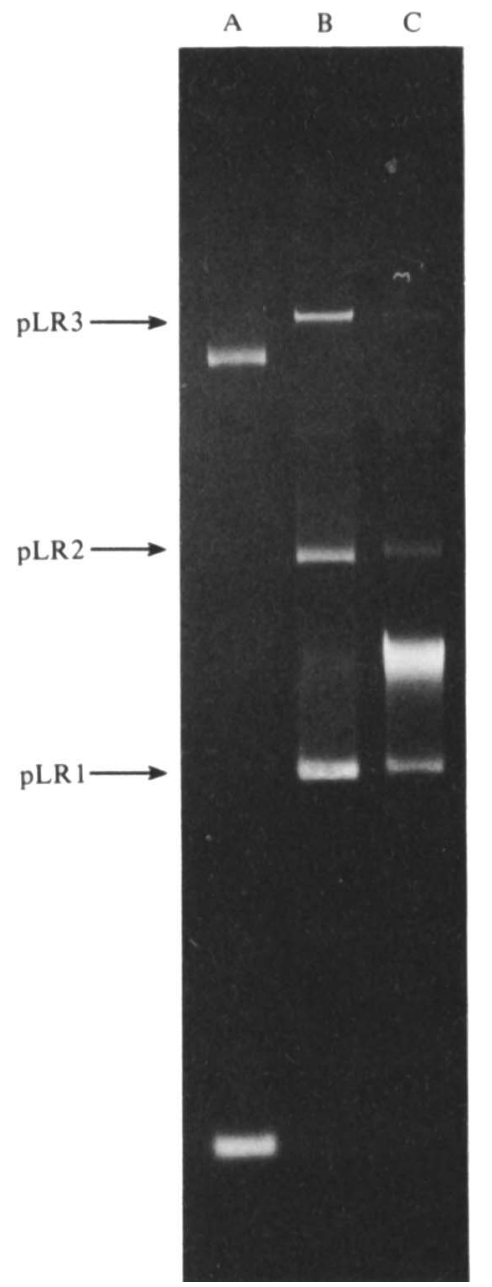

Fig. 1. Agarose gel electrophoresis of plasmid DNA from $M$. avium intracellulare LR25. Agarose $(0.8 \%, \mathrm{w} / \mathrm{v})$ was dissolved in Tris/acetate buffer. A, marker plasmids R222 (mol. wt $70 \times 10^{6}$ ) and pSC $101\left(6 \times 10^{6}\right)$ isolated from Escherichia coli; B, purified plasmid DNA from strain LR25; C, crude polyethylene glycol precipitated DNA from strain LR25.

\section{Restriction-modification system in strain LR25}

In an effort to develop a phage typing scheme for $M$. avium intracellulare we tested a variety of mycobacteriophages for their ability to infect strains of this group. All the phages studied had been propagated in $M$. smegmatis ATCC 607. Phage JF2-607 when plated on strain LR25 gave an efficiency of plating of $10^{-4}-10^{-5}$ compared with plating on $M$. smegmatis. One of the rare plaques on strain LR25 was picked and propagated on strain LR25 to obtain a high-titre stock. This phage (JF2-LR25) plated with approximately equal efficiency on strain LR25 and on $M$. smegmatis. Propagation of phage JF2-LR25 on $M$. smegmatis, which is not a selective procedure, yielded phage that again plated with low efficiency on strain LR25. This cycle was repeated several times with the same result. These results indicate the presence of a host R-M system in strain LR25 and the absence of such a system in our strain of $M$. smegmatis. We have identified several other $M$. avium intracellulare strains that are sensitive to phage JF2; phage propagated in these strains is restricted by strain LR25. 


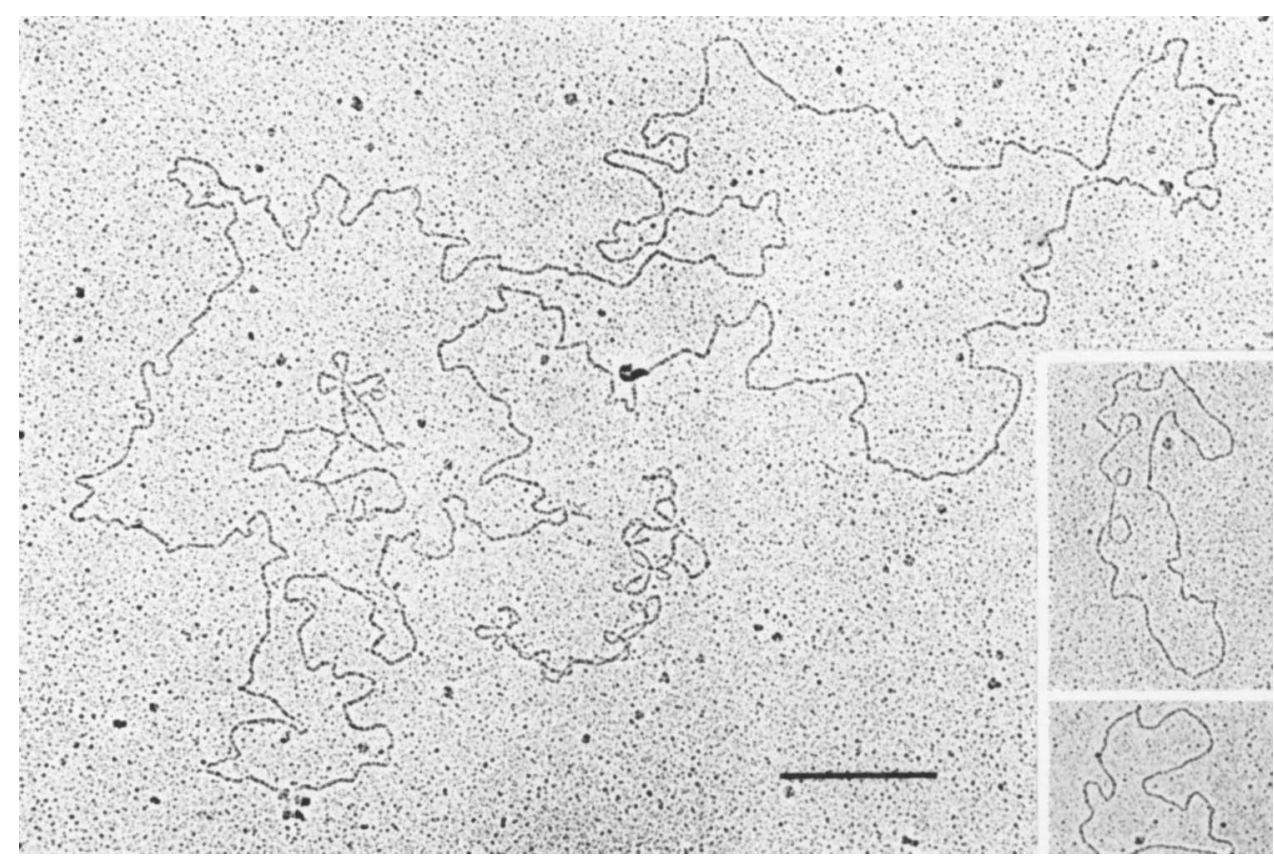

Fig. 2. Electron micrographs showing representative molecules of the three plasmids isolated from $M$. avium intracellulare LR25. The bar marker represents $0.1 \mu \mathrm{m}$.

Table 1. Effect of the propagating host on the efficiency of plating of phage JF2

Phage JF2 was propagated on the hosts indicated and then its titre was determined on the strains listed. The highest titre was always obtained with $M$. smegmatis, and this was taken as an efficiency of plating of 1 .

\begin{tabular}{|c|c|c|c|}
\hline & \multicolumn{3}{|c|}{ Efficiency of plating } \\
\hline Plating cells & ATCC 607 & $\begin{array}{l}\text { Propagating host: } \\
\text { LR25 }\end{array}$ & LR 163 \\
\hline $\begin{array}{l}\text { M. smegmatis ATCC } 607 \\
\text { M. avium LR25 } \\
\text { M. avium LR } 163\end{array}$ & $\begin{array}{c}1 \\
10^{-4} \\
0.5\end{array}$ & $\begin{array}{l}1 \\
0.5 \\
0.5\end{array}$ & $\begin{array}{r}1 \\
10^{-4} \\
0.5\end{array}$ \\
\hline
\end{tabular}

\section{Isolation and characterization of a cured strain}

To determine whether the R-M system of strain LR25 was plasmid-coded, strain LR25 was grown in the presence of acriflavine to eliminate the plasmids and tested for sensitivity to phage JF2-607. Forty isolates were tested, and one was found to be sensitive. This strain, designated LR 163, did not restrict phage JF2-607, and phage JF2 propagated in strain LR 163 was restricted by strain LR25 (Table 1). Therefore, strain LR163 is both restriction and modification deficient. In repeated attempts, we have failed to detect plasmid DNA in strain LR 163, and we believe that all three plasmids have been lost. We have repeated the curing procedure and have isolated two additional R-M-deficient strains. Unfortunately, both of these have also lost all three plasmids. We conclude that the $\mathbf{R}-\mathbf{M}$ system is plasmid-coded, but we do not know which of the three plasmids is involved.

Strain LR163 was found to be sensitive to phages AN9, AN3, D302, VA6, JF1 and JF3, all propagated on $M$. smegmatis. None of these phages formed plaques on strain LR 25 even when high numbers of phage were plated, and we were unable to isolate modified phage. 
Although it is possible that restriction of these phages by strain LR25 is more efficient than restriction of phage JF2, it is more likely that some plasmid-coded function in addition to restriction is involved.

Strain LR25 was sent to the clinical laboratories at National Jewish Hospital for identification. It did not react in a characteristic way with the available antisera and could not be serotyped. However, analysis of cell wall lipids by thin-layer chromatography (Brennan et al., 1978) showed peptidoglycolipids characteristic of serotype 6 strains. Subsequent analysis indicated that strain LR 163 was identical to the parent strain LR25 in this regard.

\section{Demonstration of modified phage in soil}

In an attempt to isolate phages specific for $M$. avium intracellulare, a number of samples of barnyard soil were tested for the presence of phage by plating on $M$. smegmatis and various $M$. avium intracellulare strains. Surprisingly, one sample from a chicken run gave plaques on strain LR25 with a titre about the same as that on $M$. smegmatis. Several isolated plaques on strain LR25 were picked, and the phage were propagated on strain LR25 and $M$. smegmatis. Phage propagated on strain LR25 plated with equal efficiency on both strains, whereas phage propagated in $M$. smegmatis gave an efficiency of plating of $10^{-5}$ on strain LR25. This indicates that the phage is sensitive to restriction by strain LR25 and the particles in the original soil sample were modified. This new phage was designated JF4.

\section{A chromosomal $R-M$ system}

We detected an R-M system in strain LR131 (serotype 9) using phage JF1. As was the case with strain LR25, phage propagated in $M$. smegmatis was restricted by strain LR131, whereas phage propagated in strain LR 131 plated with approximately equal efficiency on strain LR131 and M. smegmatis. Repeated analyses indicated that strain LR131 did not carry plasmids, and we conclude that the R-M system in strain LR131 is chromosomal. Unfortunately, strain LR131 was resistant to phage JF2, and strain LR25 was resistant to phage JF1; therefore, we have not determined whether the specificities of the R-M systems in the two strains are the same.

\section{DISCUSSION}

Our results clearly indicate the presence of $\mathbf{R}-\mathbf{M}$ systems in two strains of $M$. avium intracellulare, although we have not directly demonstrated restriction-modification at the level of DNA. In strain LR25 the R-M system is apparently plasmid-coded, whereas in strain LR 131 the R-M system is a chromosomal function. Phage propagated in these strains were not restricted in M. smegmatis ATCC 607, indicating the absence of an alternate restriction system in this strain. Jones \& Greenberg (1977) demonstrated R-M systems in Mycobacterium chelonei and $M$. smegmatis using phage $\phi 630$. In this instance both strains exhibited restriction. Evidence for restriction in Mycobacterium tuberculosis has also been reported, and it was proposed that this could account for the phage types in these organisms (Rado et al., 1976).

The demonstration of an R-M system in a strain that we knew to carry plasmids prompted us to attempt curing. The isolation of an $\mathbf{R}-\mathbf{M}$-deficient strain following acriflavine treatment and the subsequent demonstration that the strain was free of plasmids is strong but not conclusive evidence that the $\mathbf{R}-\mathbf{M}$ system is plasmid-coded. Direct proof as well as identification of the plasmid involved would be provided by transfer of the marker to another strain. However, preliminary experiments with LR25 indicate that infection with unmodified phage kills the cells, and therefore the restriction phenotype probably cannot be directly selected.

The presence of R-M systems would presumably be of no clinical significance but would be a useful marker for studies of epidemiology. We have tested over 60 strains of the $M$. 
avium complex and found that almost one-half were sensitive to phage propagated in $M$. smegmatis (unpublished observations). The phage-resistant strains were also resistant to phage modified by propagation on strains LR25 and LR131. This suggests that R-M systems may not be common in these organisms. However, if the $\mathbf{R}-\mathbf{M}$ systems are plasmid-coded they may be lost on repeated transfers, and studies of freshly isolated strains may give different results.

Regardless of the importance of R-M systems, the marker allowed us to isolate a cured derivative of strain LR25. By comparing strain LR25 and strain LR 163 it should be possible to identify other plasmid-coded functions, and we have already noted a difference in phage sensitivity which appears to be unrelated to restriction. We have shown that mycobacterial plasmids can be characterized by physical means, and these plasmids will facilitate the development of mycobacterial genetics.

This paper is dedicated to the memory of James K. Fitzhugh.

We thank Anna Tsang and Patrick Brennan for serotyping and lipid analysis of our strains. This work was supported in part by Public Health Service Grant RO1 GM2 1446 to M.D.C. and by Veterans Administration Research Funds, Project no. 0834-004.

\section{REFERE N C ES}

Brennan, P. J., Souhrada, M., Ullom, B., McClatchy, J. K. \& Goren, M. B. (1978). Identification of atypical mycobacteria by thin layer chromatography of their surface antigens. Journal of Clinical Microbiology 8, 374-379.

Crawford, J. T. \& Bates, J. H. (1979). Isolation of plasmids from mycobacteria. Infection and Immunity 24, 979-981.

Davis, R., Simon, R. \& Davidson, N. (1971). Electron microscope heteroduplex methods for mapping regions of base sequence homology in nucleic acids. Methods in Enzymology 21, 413428.

Johnson, P. H. \& Grossman, L. I. (1977). Electrophoresis of DNA in agarose gels. Optimizing separations of conformational isomers of double- and single-stranded DNAs. Biochemistry 16, 42174224.

Jones, W. D., JR \& Greenberg, J. (1977). Host modification and restriction with a mycophage isolated from a pseudolysogenic Mycobacterium chelonei. Journal of General Microbiology 99, 389-395.

Meyers, J. A., Sanchez, D., Elwell, L. P. \& FAlkow, S. (1976). Simple agarose gel electrophoresis method for the identification and characterization of plasmid deoxyribonucleic acid. Journal of Bacteriology 127, 1529-1537.

Rado, T. A., BATES, J. H. \& Fitzhugh, J. K. (1976). Evidence for host-dependent modification and restriction of bacteriophage DNA in Mycobacterium tuberculosis. Journal of General Virology 30, 91-97. 